\title{
À PROPOS DE ON EN FRANÇAIS: RESTRICTIONS DISCURSIVES ET INTERPRÉTATION
}

\author{
Francine Mazière
}

RESUMO: A análise do discurso, em sua exigência de leitura da história, apoia-se sobre a língua. Este trabalho questiona diacronicamente a constituição da interpretação lexical do pronome on, em francês, através de sua fixação morfosemântica e discursiva nos primeiros dicionários. Analisando os discursos sobre on $e$ os discursos produzidos com on, percebe-se uma semantização histórica da partícula, legível no dicionário, instrumento lingüístico cujo objeto não seja, talvez, a língua da gramática, mas a da normatividade cultural.

PALAVRAS-CHAVE: análise de discurso, dicionários, história da língua, interpretação lexical, classes de referência.

Ce travail porte sur une unité lexicale, le pronom dit indéfini on, dont l'interprétation est problématique en français, où il ne connaît pas la même dispersion qu'en portugais.

On est intéressant en analyse de discours à plusieurs titres:

1. pronom non anaphorique, il construit ses ambiguités et son interprétation dans le texte;

2. pronom indéfini non anaphorique dans l'intradiscours, il peut, par effet de l'interdiscours, représenter un préconstruit;

3. parfois appelé personnel et toujours en position de sujet, il peut, plus aisément que d'autres, représenter toutes les personnes du verbe. Il participe à l'obligation de repenser l'analyse énonciative, et plus précisément benvenistienne, des personnes et, en particulier, les notions d'inclusion et d'exclusion.

Une analyse de discours devrait nous permettre d'interroger plus particulièrement les conditions de son instabilité sémantique et les limites de cette instabilité à travers le double problème des conditions de substitutions interdiscursives et des restrictions des sous-ensembles discursifs autorisés.

Dans un premier temps, je vais donc m'efforcer d'exposer l'originalité de 
ce lexème, supposé dérivé du GN l'homme. J'exposerai ensuite la conscitution de son sémantisme à partir de définitions en langue et d'emplois discursifs. Le corpus me sera directement fourni par l'outil linguistique qui conjoint langue, discours sur la langue et discours historicisé: le dictionnaire. J'aurai recours, pour analyser sa constitution, aux deux premiers dictionnaires monolingues français, parus à la fim du XVII ${ }^{\mathrm{e}}$ siècle, le Dictionnaire Universel d'Antoine Furetière (1690) et le Dictionnaire de l'Académie (1694).

\section{CONSTITUTION D'UN PRONOM-LEXÈME}

\section{De l'homme dit à on dit}

Em règle générale, tous les dictionnaires français, y compris les plus récents - le Lexis (LAROUSSE, 1985), hérité des travaux du linguiste Jean Dubois ou le Dictionnaire historique de la langue française (LE ROBERT, 1992) - donnent à on l'origine homme. Le point curieux de l'affaire est sans doute le grand rôle joué par l'orthographe dans cette attribution. Littré, grand lexicographe français du XIX ${ }^{\mathrm{e}}$ siècle, donne la forme om comme première attestation de on dans le Serment de Strasbourg, premier texte répertorié en français $\left(\mathrm{XI}^{\mathrm{e}}\right.$ siècle), mais retient cette même graphie en provençal pour homme.

A partir du XVI ${ }^{\mathrm{e}}$ siècle, les orthographes hom, l'hom et on, l'on, entrent en concurrence. Pour Robert Estienne, qui produit la première mise en ordre alphabétique du français, en 1539, seul hom, (pour homme, ajoute-t-il) est correct, mais il donne ce mot comme manière française d'exprimer avec um verbe actif les verbes impersonnels de voie passive du latin. Curritur: Hom cueurt. id est homo currit. L'équivalence sémantico-syntaxique est ainsi posée, qui sera répétée dans les dictionnaires bilingues français-latin du siècle suivant, et servira à alimenter les discussions sur la présence et l'absence d'une trace de déterminant (l'on).

Cependant, dès le début du XVII ${ }^{\mathrm{e}}$ siècle, Nicot, fin observateur de langues (il est l'auteur de dictionnaires plurilingues et le continuateur de Robert Estienne pour le dictionnaire français-latin) ${ }^{1}$, met en concurrence orthographique on $=h o m$ avec une spécificité d'usage. L'orthographe on s' impose, sans variation possible, "devant des verbes comme dire, connaître et [autres verbes] semblables" (NICOT, Thrésor de la langue française, 1606). Cette curieuse remarque sur l'usage écrit va être le point de départ de l'analyse que je propose.

Quarante ans plus tard, Vaugelas, célèbre auteur de remarques sur la langue française, admet l'orthographe on d'emblée et rejette la filiation par omnis au profit de homo. Il s'appuie pour cela sur l'abréviation de huomo en huom (d'où hom) chez les poètes italiens, devant un verbe commençant par une consonne

\footnotetext{
${ }^{1}$ Pour l'anecdote, il a été en poste à Lisbonne, où il a eu le loisir d'apprécier le vocabulaire portugais de la navigation, et on le soupçonne d'être à l'origine de l'inflation des termes de marine dans la lexicographie française.

120
} 
(huom teme). Cependant, Vaugelas semble très prudent, comme l'atteste sa formulation du parallèle homme/on: "comme si on dit voulait dire homme dit, et l'on dit voulût dire l'homme dit'.

Peu importe cette prudence, les discussions sur l'origine ne nous intéressent ici que par les collocations sur lesquelles s'appuie la discussion. On voit que, chez Vaugelas comme chez Nicot, on fonctionne en emploi privilégié avec le verbe dire. C'est le même exemple encore qu'on trouve dans le premier dictionnaire monolingue français, le Furetière $^{2}$, dans une remarque sur le site discursif donné comme origine attestée: l'huom'dice en italien. L'article se poursuit ainsi: "Borel dit avoir vu d'anciens actes des crieurs publics qui commençaient leurs publications par cette formule, hom fait scavoir, pour dire on fait scavoir".

On dit, on fait savoir, on connait que, parait ainsi être la distribution canonique de $o n^{3}$. Tout se passe comme si un site discursif, au cceur du ça se dit, ça se sait, se connaît, autrement dit, la doxa discursive, avait contribué de façon privilégiée à produire la grammaticalisation pronominale du concept d'homme, non comme animal supérieur, ni même comme animal raisonnable mais comme diseur (je propose ce néologisme à titre de terme métalinguistique) parce que porteur d'un savoir. Comme porteur de parole informante.

Dans cette étude, à partir du double fonctionnement de on comme collectif et comme personnel spécifié, répertorié pour la première fois par le premier Dictionnaire de l'Académie ${ }^{4}$, nous retiendra sa capacité à contraindre l'interprétation ${ }^{5}$ par le seul jeu des substitutions dans les usages attestés du XVII ${ }^{\mathrm{e}}$ siécle $^{6}$.

\footnotetext{
${ }^{2}$ Le Dictionnaire Universel d'Antoine Furetière est souvent considéré comme le premier dictionnaire monolingue français, malgré la sortie, 10 ans plus tôt, d'un recueil monolingue rédigé par Richelet. C'est un ouvrage à tendance plus encyclopédique que le Dictionnaire de l'Académie. Furetière est un académicien dissident qui publie avant et contre l'Académie.

${ }^{3}$ Cela me signifie nullement que on fait/on donne... ne soient pas attestés, mais que les instruments d'enregistrement de la langue et des discours raisonnent essentiellement à partir de on dit comme place où homme s'est prioritairement fixé en on.

${ }^{4}$ Le Dictionnaire de l'Académie est publié à Paris em 1694. Bien que souvent critiqué pour son purisme, ce dictionnaire me semble le seul dictionnaire de langue édité en europe au XVII ${ }^{\mathrm{e}}$ siècle, et inégalé pour le français jusqu'aux plus récentes avancées de la lexicographie contemporaine. C'est donc un outil linguistique de premier ordre, un outil rare et original, fondateur de langue, voire d'unités linguistiques, et qui, nous donnant le recul de l'histoire, nous permet de mieux saisir les enjeux discursifs à l'œuvre.

${ }^{5}$ Cette expression est empruntée à Marandin, 1993.

${ }^{6}$ Je renvoie, pour des analyses plus sociolinguistiques, aux articles de Josiane Boutet et Catherine Viollet dans LINX n. 18, 1988, Analyse grammaticale de corpus oraux, coordonnés par F. Gadet et F. Kerleroux. Ces articles abordent entre autre deux aspects de la particule qui ne sont pas retenus ici: que on puisse remplacer toutes les personnes, et donc contrevenir au partage selon Benveniste entre personne et non-personne, que dans som rôle de $3^{\mathrm{e}}$ personne exclusive, il ne puisse être anaphorique.
} 


\section{Le problème du collectif et de la personne}

L'article du Dictionnaire de l'Académie se présente en deux parties:

Particule collective qui tient lieu de pronom personnel indéfini \& qui ne se joint jamais qu'avec la troisième personne singulière des verbes: on dit que, on raconte, on fait la guerre [...] qu'en dira-t-on?

Il signifie quelquefois simplement Quelqu'un ou quelqu'une. on lay a confié..., on luy a écrit...

On notera: la distinction entre collectif et spécifique (en substitution à quelqu'un), qui sera retrouvée par nos dictionnaires contemporains ${ }^{7}$. Mais il y a plus étonnant. Ce dictionnaire a la particularité de donner la langue à partir de l'usage quotidien "des honnêtes gens, des poètes et des orateurs", et cet usage, à partir des collocations, c'est à dire des sites discursifs, des dépendances mutuelles de termes les plus répandus. Il est intéressant de noter que dans la première définition, construite sur les adjectifs collectif et personnel indéfini, et suivie de formes où prime le dire, on fonctionne comme seule particule préverbale, tandis que la deuxième définition (quelqu'un) est suivie d'expressions où on s'oppose à un autre pronom, lui et se joint à tout verbe actif. On ne peut plus clairement et plus grammaticalement en termes de distribution séparer le spécifique $(o n+l u i+$ verbe actif) du collectif premier (on $+\mathrm{v}$. dire). La distribution trie le sens en réduisant l'indécidabilité énonciative. Reste que collectif est glosé par personnel indéfini. La définition-indéfinition discursive de ces personnes prises - par leur dire, et pour leur dire - dans le collectif, et les conséquences pour l'analyse des dires, sera analysée à la suite.

\section{Quelques propriétés linguistiques de on}

De ce détour par l'histoire de la langue on retiendra, dans le passage du nom au pronom, la fixation d'une morphologie insensible aux variations de genre et de nombre. On est le dérivé d'une abstraction de homme plus que d'un concret homme. Il est le plus neutre des représentants (ce excepté) en ce sens qu'il neutralise genre et nombre. D'autres indéfinis français ne vont pas jusque là: tout (e, es).

Il y a donc fonctionnement autonome du mot comme (1) pouvant appartenir à toutes les personnes, (2) de sens global (pour homme disant-pensantfaisant), (3) jouant sur singulier et pluriel, masculin et féminin, c'est à dire réalisant un lieu de neutralisation de ce que beaucoup d'anaphoriques non seulement ne neutralisent pas mais ne peuvent pas ne pas indiquer (cf. ils, elles, celui-ci, celle-ci,

\footnotetext{
${ }^{7}$ Furetière, contemporain de l'Académie, était plus confus et on peut penser qu'il est à l'origine de l'analyse souvent répétée d'un sémantisme de on incertain et indefini. Je donne l'article de Furetière: "substantif masculin, qui par corruption s'est réduit en une particule indéclinable qui se joint avec les verbes impersonnels, \& qui a la force d'un nom collectif, ou de plusieurs personnes incertaines ou indéfinies" Pour une analyse plus développée, cf. Mazière 1994.2.

${ }^{8}$ On entend par là le personnel politique, celui qui peut haranguer le Roi.

122
} 
son, sa, ce cette...). Nous tenons là une donnée de langue intéressante car une unité neutralisée est bonne à investir de sens. Avec on, toutes les neutralisations semblent laisser des places pour redévelopper l'effacé. Ces possibilités grammaticales, ou rhétoriques (syllepses), contraignent les lexicographes à signaler quelques attractions de genre (on est coquette ou on n'est pas) ou de nombre (ici, on est tous égaux) au niveau des participes passés ou des attributs.

$\mathrm{Au}$ plan discursif qui nous intéresse, cette instabilité revient à l'interrogation: Qu'en est-il de la place de la personne dans le collectif? Autrement dit, de la spécification du/des collectif(s)? Et, en particulier, qu'en est-il de ce collectif, qui fonctionne à la fois comme nous et comme ils, dans l'inclusion et dans l'exclusion?

\section{ANALYSE DISCURSIVE}

C'est dans l'article de dictionnaire que nous examinerons comment le discours rapporté, comme le pré-asserté, s'effacent, s'oublient, dans et par le on et, par cette opération, donnent sens à la notion de collectif.

Comme le dit Bayle, préfaçant Furetière, le dictionnaire est plein de ces petits mots: on dit... Il n'est pas indifférent que ce soit dans un dictionnaire à tendance encyclopédique comme le Furetière que soit signalé le phénomène. De façon plus générale, on sert au lexicographe pour rédiger son article, de la définition à l'exemple forgé. Le texte de l'article de dictionnaire, lu comme un discours et un dispositif interdiscursif ${ }^{9}$, est un site privilégié pour analyser l'étonnante ductilité discursive de la particule. Je prendrai mes exemples dans le Furetière et le Trévoux ${ }^{10}$.

\section{On joue un rôle métadiscursif dans la mise en forme de l'article}

\section{Comme encadrement des équivalences d'ordre discursif}

On dit... pour dire: Cet encadrement est, chez Furetière, la forme la plus générale pour introduire des sous-entrées dans l'article. EIle est discursivement primordiale car elle repère dans la langue les locutions, les collocations du prêt à parler (COLLINOT; MAZIÈRE, 1987). Elle répertorie la convention, les dires possibles, autorisés, dans la masse des sens admis par la communauté.

ONCLE: [...] On dit proverbialement la vigne à mon oncle pour dire la

\footnotetext{
${ }^{9}$ Toutes les études signalées en notes, qu'elles soient co-signées avec André Collinot ou avec Jacqueline Léon, posent l'article de dictionnaire comme un discours tenu sur le sens, dans une continuité allant de l'énoncé définitoire à l'exemple. L'article de dictionnaire étant rédigé en langue naturelle, il ne livre pas seulement du sémantique mais aussi du discursif.

${ }^{10}$ Dictionnaire français-latin inspiré des dictionnaires de Furetière et de l'Académie et réédité, avec des augmentations, durant tout le XVIII ${ }^{\mathrm{e}}$ siècle. Nombreuses passes d'armes avec l'Encyclopédie de Diderot.
} 
plus proche du village

Ce premier on, le plus simple, pose une communauté linguistique homogène, en contradiction flagrante avec tout ce qu'on sait de la situation linguistique de l'époque ${ }^{11}$. Cette fiction est nécessaire à la création de 'la langue française' comme objet de référence (COLLINOT; MAZIĖRE, 1993). C'est un coup de force normatif qui va durablement modifier la situation linguistique nationale. Le dictionnaire fonctionne bien alors comme un "outil linguistique" (AUROUX, 1992).

\section{Comme spécification des discours}

On dit... en (Physique)/ on dit en parlant de: forme qui sous catégorise les locuteurs en fonction des lieux de l'usage langagier, et prépare l'introduction de la technique des domaines d'emplois en lexicographie (MAZIĖRE, 1990).

FIBRE [...] on donne aussi ce nom en Physique à... En l'usage commun, $o n$ ne le dit que des... Kepler... ${ }^{12}$

La possibilité de lire l'article de dictionnaire comme une répartition des dires dans la langue nationale est ici flagrante. On est toujours collectif, mais tire ses restrictions de sens du domaine d'emploi, jusqu'à l'extrême spécificité, le nom propre.

\section{On est en usage discursif dans l'énoncé définitoire}

Si l'on passe de l'organisation de l'article dans son ensemble, avec ses rubriques obligées dont la lecture est guidée par la typographie, à la rédaction de la seule définition, la distribution énonciative devient plus complexe: le lexicographe est un porte plume de la doxa sur le sens de tel ou tel mot. Mais si l'on pose des lieux différents du dire le sens dans une société donnée, parfois il exhibe, le plus souvent il efface, les énonciateurs du sens, experts ou anonymes, singuliers ou pluriels.

Nous examinerons ces coutures discursives en allant du discours rapporté, très fréquent dans les définitions de type encyclopédique, à des discours étranges, étrangers au lexicographe, dont la présence insiste comme par hasard, et nous conduit à nous interroger sur la nature divisée du locuteur collectif.

\section{On dit (que): Le discours rapporté dans les définitions de type encyclopédique}

ZABACHE. La mèr de Zabache, [...]. C'est um lac situé sur les confins de l'Europe \& de l'Asie, entre la petite Tartarie \& la Circassie. On lui donne six cent mille pas [...] mais on assure qu'il a si peu de fonds qu'il

\footnotetext{
${ }^{11}$ Sur les dix-sept millions d'habitants que compte la France à cette époque, trois parlent français. Les frontières sont partout, linguistiques et législatives. L'unification nation/langue nationale, qui fait de la France un exemple singulier en Europe, date de la période révolutionnaire, fin de XVIII ${ }^{\mathrm{e}}$ siècle.

${ }^{12}$ C'est moi qui souligne en italique. 
ne peut $[\ldots]$.

Bien des connaissances, quand elles ne sont pas théologiques, sont à cette époque vécues comme peu sures. Le lexicographe conserve à l'article de dictionnaire son caractère de vulgarisation en ayant recours à des sources dont il ne donne pas toujours les références. Ce type de discours est donc extrêmement fréquent dans Furetière et Trévoux (définitions de choses, pour reprendre la terminologie qui sera celle de Diderot, liées à l'état encyclopédique des connaissances), au contraire de l'Académie, strictement linguistique (définitions de mots), qui n'admet ni les noms propres, ni les mots spécialisés. Ici, dans une sorte de connivence énonciative, on lui donne, on assure, introduisent le discours d'expert sans référence systématique à/aux experts ${ }^{13}$ et même parfois dans une parfaite disjonction avec les experts:

GIRAFE: Animal farouche dont plusieurs Auteurs font mention mais que personne n'a vu

Ici la communauté culturelle, non seulement n'a pas vu, mais semble prêt à exclure d'entre les personnes ceux qui racontent que la girafe existe.

Soit maintenant l'entrée ZAARA (nous disons et écrivons aujourd'hui Sahara):

ZAARA, s.m. ou le DÉSÈRT. Nom d'une grande région de l'Afrique. [...] Il est divisé en sept Royaumes ou Déserts, que l'on rencontre em cet ordre [...]. On y trouve de vastes campagnes de sables mouvants, \& dangereuses pour les Voyageurs, ce qui a obligé les Arabes à lui donner le nom de Mèr de sable. MATY

Les deux on désignent sans doute plus le voyageur que le lexicographe et ses lecteurs. Cependant tous se retrouvent liés en une même communauté face à un nouvel autre: les Arabes. Même si le discours rapporté encyclopédique (renseignement sur la chose) est souligné par um nom d'auteur (Maty), une nouvelle partition se dessine par la désignation d'un complémentaire au on de la communauté culturelle. Ce sont les Arabes, comme actants-source (curieusement contraints!) du nom-entrée, qui donnent au on du lexicographe, par effet de partition, une appartenance culturelle. Le collectif, sens le plus attesté de on dans les premiers dictionnaires, a donc des frontières, contrairement à l'universel. Je reviendrai en 2-3 sur l'idée d'universel empirique dans la définition de on.

Or, cette seconde valeur de on, em co-occurrence avec un complémentaire, dont on admet aisément qu'elle soit fréquente dans l'énoncé encyclopédique, est, paradoxalement en apparence, semblable à celle de on en énoncé définitoire de langue, c'est à dire quand il mime l'universel définitoire. C'est ce qu'on peut voir à la suite.

\section{Le discours définitoire prend sens par l'exemple: la doxa est une construction}

Quand on traite l'article de dictionnaire comme un discours suivi, on

${ }^{13}$ La citation régulière des sources, la liste des auteurs et des ouvrages consultés, seront une conquête de la lexicographie encyclopédique du XIX ${ }^{\mathrm{e}}$ siècle, qui n'aura plus recours à cette forme d'indetermination. 
repère que l'emploi de on collectif, en définition, ne prend sens que dans le contraste avec un sous groupe nommé, substitut de on, dans les exemples forgés par le lexicographe, c'est à dire dans les paroles correspondant au plus banal, ou au plus adéquat de l'emploi:

FICTION: [...] Inventions poétiques qu'on se met dans l'esprit. Les Anciens avaient [...]. Ce malade se met dans l'esprit toutes sortes de fictions.

L'exemple vient donner sens au on collectif de la définition dont il reprend jusqu'à l'ordre des mots. On se met dans l'esprit,/ce malade se met dans l'esprit. Sans doute le remplacement de ce malade par Pierre ferait-il perdre toute raison d'être à l'exemple, en n'éclairant pas de qui, au sens de de quel type de personne, on peut dire qu'il se met des fictions dans la tête. On voit que l'exemple n'est pas un quelconque usage attesté. En reprenant la syntaxe de la définition, il fait plus qu'exemplifier. Il fait sens en décomposant on en ses substitutions possibles, en ébauchant les limites d'une classe de référents.

On pourrait multiplier les citations:

FICHE: pièce qu'on fait entrer [...], qui sert au maçon pour faire entrer [...].

FIANCE ${ }^{14}$ : assurance qu'on avait de la fidélité de quelqu'un, Le Prince avait grande fiance à son écuyer.

Deux rôles (prince, écuyer) se substituent à on et quelqu'un. Il y a donc déroulement d'un paradigme ordonné pour construire l'usage discursif du sens ${ }^{15}$. Ici, le maçon pour la fiche, le prince pour la fiance, sont une autre façon de dire le domaine d'emploi des mots mais, aussi de valider les termes classificatoires de la définition, outil d'une part, fidélité et donc droit personnel et allégeance d'autre part. Tous les sujets humains ne se valent pas, ne sont pas interchangeables, ne forment pas un paradigme ouvert où tout pourrait se dire. L'espace de description linguistique que constitue le dictionnaire est en cela bien différent de la grammaire où Luc et Marie tiendraient lieu de maçon et de prince. La grammaticalité et l'acceptabilité n'y sont pas de même ordre. Le rapport à la langue non plus. Ce qui explique peut-être la rareté des dictionnaires de langue au profit des dictionnaires encyclopédiques, plus ouvertement pris dans l'ordre du discours. Les dictionnaires de langue sont un objet théorique et empirique difficile.

\section{Mais qui n'est pas on? ou comment circonscrire le collectif social?}

Il ressort d'une étude de modélisation de définitions de noms d'instruments dans le Furetière, que, très majoritairement, quand la relative déterminative qui constitue le cœur de la définition de nom est en $q u e^{16}$, son sujet

\footnotetext{
${ }^{14}$ Mot vieilli pour confiance.

${ }^{15}$ Une étude sur les restrictions d'emploi des noms dans les places vides ménagées par la définition a été proposée dans Collinot/Mazière, 1984.

${ }^{16}$ Les noms, dans les dictionnaires classiques, sont en géneral définis ainsi: nom-entrée: $\mathrm{N}$ (générique) qui $\mathrm{V} / \mathrm{N}$ que $\mathrm{P}$ (où $\mathrm{P}$ contient un $\mathrm{SN}$ sujet de référence autre que le génerique. Ex: pigeon: oiseau qui 126
} 
est de la forme on. (cf. supra FICTION, FICHE, FIANCE) (LÉON/MAZIÈRE, 1990). Cependant il y a des exceptions: sur 40 entrées constituant le petit corpus modélisé, 21 présentaient une définition en $N$ que, et 14 seulement avaient on pour sujet. Les autres sujets étant la nature (2 fois), les vieillards, les gens infirmes, les Prétendus Réformés, le peuple, les Mahométans.

L'énoncé définitoire liste donc en clair, quand il cite une catégorie d'humains, ceux qui se distinguent de la communauté linguistique, de la collectivité, par des mots qui désignent leurs particularités. Dans notre courte liste, ce sont les vieux, les malades, le peuple et les non-catholiques. Encore une fois se marque une délimitation des dires, des frontières au collectif, une communauté humaine qui n'est pas l'humanité universelle qui sera pensée au XIX ${ }^{\mathrm{e}}$ siècle. Mais ici, il ne s'agit plus de délimiter des domaines d'emploi mais des spécificités de désignation. Le dictionnaire fonctionne comme un corpus de discours historiquement déterminés, une archive qui enregistre la doxa et, marquant les discours rapportés dans les textes les plus nettement encyclopédiques, laisse ouvert le jeu interprétatif dans les définitions plus linguistiques. L'exemple est alors central pour dire le sens de la définition. (COLLINOT; MAZIÈRE, 90).

\section{Lecture du dictionnaire}

Ainsi, sans qu'il soit besoin d'avoir recours à des analyses d'entrées idéologiquement marquées, la forme syntaxique de la définition et l'enchaînement intradiscursif avec l'exemple qui met le mot en emploi, installent des procédures de lecture de tout article de dictionnaire.

Pour le lexème on qui nous intéresse ici, la rédaction de la définition est donc bien un site discursif privilégié pour lire le jeu de l'indéfini et du spécifique comme jeu des pluriels dans un singulier culturel purement idéologique. Elle permet en particulier de noter la permanence, au XVII ${ }^{\mathrm{e}}$ siècle, d'une frontière discursivement repérable entre on et d'autres, la particule intégrative jouant comme réacteur. Elle oblige à dégager son complémentaire au moment même ou elle se positionne dans le discours comme collectif. Rien de vague dans cette particule, contrairement à ce que posent certaines définitions contemporaines dans leur définition de $o n^{17}$, mais du sens qui ne se construit que dans de l'interdiscours, à l'intérieur du domaine des substituables discursifs, et dans cette co-occurrence des personnes, mise en scène dans les exemples du premier Dictionnaire de l'Académie.

Si nous revenons sur la formulation du Dictionnaire de l'Académie, nous pouvons maintenant mesurer toute la pertinence d'un dictionnaire de langue travaillant en parfaite synchronie, à partir de collocations. Ce dictionnaire est le seul (MAZIÈRE, 1994) à poser le collectif en surplomb sémantique: "Particule

vit... / oiseau qu'on élève pour... Collinot/Mazière 1987, Mazière 1987, 1989.

${ }^{17}$ Le terme vague s'introduit dès Littré, au XIX ${ }^{\mathrm{e}}$ siècle: "Il indique de manière générale ou vague les gens, les personnes." 
collective qui tient lieu de pronom personnel indéfini”.

Mais si l'Académie a l'avantage d'une double définition en langue, reposant sur la distribution, Furetière dit le même sémantisme du côté encyclopédique: "qui a la force d'un nom collectif". Cela permet à la lexicographie du XVII ${ }^{\mathrm{e}}$ siècle tout le jeu du rôle social, où les exclus de la communauté langagière se listent, prêts, dans une autre configuration discursive, plus régionaliste, à s'interpréter comme personnes spécifiques, et, comme telles, à pouvoir être vilipendées ou valorisées.

Cette lexicographie me semble donner parfaitement à voir le travail de l'espace-temps que convoque Auroux dans sa définition de l'hyperlangue (1994). Plus généralement, les outils qui contribuent à l'histoire de la langue, ou les outils témoins et acteurs de cette histoire — grammaires, remarques, dictionnaires —, ne seraient-ils pas un lieu d'inscription non maîtrisée de ces traces discursives qui font certains sémantismes de langue, un lieu révélateur, au sens photographique du terme, de l'hyperlangue?

En effet on mesure, par extension de l'analyse, que la notion d'universel proposée à partir de la lexicographie du XIXe siècle (on = tous les hommes, les gens), a tous les traits d'une formulation idéologique contrainte par un nouvel espace-temps, inscrite dans cette époque héritière des Lumières et de la Révolution. A titre d'exemple (mais je renvoie aussi à Littré, note 21), voici la définition donnée par une réédition de 1863 du Bescherelle, le Nouveau Dictionnaire classique de la langue française. On y lit:

"on: pr. indéf. (du latin homo, homme). Nom destiné à indiquer

l'universalité des personnes d'une manière vague et indéterminée."

On remarquera en outre le destiné à, très daté, bien différent du qui tient lieu de de la lexicographie du XVII ${ }^{\mathrm{e}}$ siècle.

\section{UNITÉ LEXICALE ET DISCOURS}

Parvenue à ce stade de la description, il me faudrait réinscrire le propos dans la problématique d'une analyse de discours à la fois sensible à la langue et à l'histoire. L'histoire de la langue a été peu sollicitée par l'AD. Cependant le problème du changement diachronique, s'il intéresse le système langue, se produit, pour reprendre Saussure, par et dans la parole. Et cette parole saussurienne, à laquelle on reproche son individualité, ne produit sans doute le changement que si elle est discours, c'est à dire dispositif général. A partir de là se pose le problème de la nature de l'unité de langue comme résultat d'une évolution historique. Y a-t-il des faits de langue pensables dans une sorte de naturalité de la langue? De quel ordre est l'instabilité de la langue? La variation dans le régulier et la normalisation de cette variation sont-ils à penser dans l'ordre du discours? L'histoire de la langue, qui a produit ces unités de langue, n'est-elle pas le lieu d'inscription des traces discursives dans la langue? 
Comme le détail, ou l'épuration, ou le plan social, au niveau lexical ${ }^{18}$, ne sortent pas indemnes de leurs usages politiques, la fabrication morpho-syntaxique de tel ou tel objet langagier (une forme de relative, les possibilités combinatoires de que, certaines anaphores, on) peut être d'ordre historique, à savoir lié, non à une évolution interne, mais à de la norme externe: une politique linguistique, la décision de décideurs intellectuels pris dans des positions idéologiques sur la langue, comme les remarqueurs du XVII ${ }^{\mathrm{e}}$ siècle en quête de clarté, ou l'Académie prise dans la définition d'une langue commune.

Entre langue et discours, le problème, du point du vue de l'analyste, ne me semble pas relever seulement d'un changement de terrain, d'une conscience historienne des productions langagières, d'une autre conception du sens, de la contrainte linguistico-discursive à l'interprétation ${ }^{19}$, mais aussi, sur des points précis, dont la forme et le nombre restent à explorer, du jeu de l'histoire de la langue dans la langue, d'une part, des possibilités de décompactification, déneutralisation exercées par le discours sur la langue d'autre part. Je pense par exemple ici à l'autre de on mais aussi à l'autre de Pierre, Paul ou Jacques que constituent le malade, le maçon et le prince, comme domaine de substitution temporalisé, le seul qui fasse sens dans une pratique langagière pensée comme discours. Autrement dit, en discours on est bien dans des histoires ("il était une fois un prince et un maçon..."), alors que la grammaire est sans histoire ("il était une fois Luc et Paul" est agrammatical). La question générale pourrait donc être: comment les catégories de temps et d'espace, qu'elles soient pensées discursivement ou pas, travaillent-elles discursivement les objets de langue historiquement constitués et analysables, c'est-à-dire dans les limites du possible à énoncer et à comprendre?

Une seconde conclusion concernerait l'objet dictionnaire comme observatoire de la langue et des discours, comme observatoire intérieur/extérieur, dont les observables ne sont ni dans, ni au bout de la longue vue de la métalangue. Ceci serait une autre étude.

\section{BIBLIOGRAPHIE}

AUROUX, S. Le processus de grammatisation et ses enjeux. In: Histoire des idées linguistiques, t.2. Introduction. Le processus de grammatisation et ses enjeux. Liège, Mardaga, 1992.

. Le langage, la raison et les normes. 1994 (à paraître).

COLLINOT, A.; MAZIÈRE, F. Langue, discours et dictionnaires. LINX, Paris,

\footnotetext{
${ }^{18}$ Je ne peux donner d'équivalences pour un Brésilien...

19 Je renvoie à Marandin 1993 pour une intérrogation magistrale sur ces question, mais aussi pour une démonstration de la spécificité des positions de l'AD dans sa relation au théorique de la linguistique.
} 
Paris-X/Nanterre, n.10, 1984.

. Un prêt-à-parler: le Dictionnaire Universel d'Antoine Furetière et sa postérité immédiate, le Trévoux. Une lecture du culturel dans le discours lexicographique. Lexicographica, Tübingen, n.3, p.51-75, 1987.

Les définitions finalisées dans le Furetière. Où il est montré que l'hétérogénéité syntaxique des définitions a du sens. In: CHAURAND, J.; MAZIÉRE, F. (org.). La Définition. Paris, Larousse, 1990.

. Une autre lecture du Dictionnaire de l'Académie. In: MOIRAND, S. et alii. (org.). Parcours linguistiques de discours spécialisés. Berne, Peter Lang, 1993.

A língua francesa: pré-construído e acontecimento lingüístico. In: ORLANDI, E. (org.). Gestos de leitura: Da história no discurso. Campinas, Ed. da UNICAMP, p.185-200, 1994.

LÉON, J.; MAZIÈRE, F. Modélisation d'énoncés définitoires à l'aide du logiciel

Déredec et analyse de discours. Litterary and Linguistic Computing, Oxford, v.5, n.1, 1990.

MARANDIN, J.J. Syntaxe, discours. Du point de vue de l'analyse de discours. Histoire, épistémologie, langage. Paris, SHESL, v.15, n.2,. 1993.

MAZIÈRE, F. Le dictionnaire, un monologue culturel? Lingua, v.804, n.2, 1987. (Cahiers de 1'Institut des langues de l'Université des Sciences Économiques. Budapest).

. O Enunciado Definidor. In: GUIMARÃES, E. (org.). História e Sentido na linguagem. Campinas, Pontes, 1989.

. Les marques de FABRIQUE. Marquage et marques dans les dictionnaires classiques (du Furetière au dernier Trévoux). Lexique, Lille, PUL, n.9, 1990. . Invention de la définition du nom abstrait dans le premier Dictionnaire de l'Académie. Actes du Colloque international Les noms abstraits. Dunkerque, Lille, PUL, 1994 (à paraître).

. On dans les dictionnaires. Faits de langue. Paris, PUF, 1994. 